\title{
Evaluation of the Accuracy and Noise Response of an Open-source Pulse Onset Detection Algorithm on Pulsatile Waveform Databases
}

\author{
Chengyu Liu ${ }^{1}$, Qiao $\mathrm{Li}^{1}$, Gari D Clifford ${ }^{1,2}$ \\ ${ }^{1}$ Department of Biomedical Informatics, Emory University, Atlanta, USA \\ ${ }^{2}$ Department of Biomedical Engineering, Georgia Institute of Technology, Atlanta, USA
}

\begin{abstract}
Zong's open-source algorithm 'wabp.c' (2003) has been widely used for onset detection of arterial blood pressure $(A B P)$ waveforms. This code was subsequently modified by Li and Clifford (2012) to avoid possible double detections in a beat cycle. However, its performance was not systematically validated, especially on a noisy pulse database. This study aimed to evaluate its detection accuracy on both clean and noisy $A B P$ pulse signals. Synchronously recorded ECG and ABP signals in two databases from the PhysioNet/Computing in Cardiology Challenge 2014 were used. Reference QRS positions were used as the benchmarks for pulse onset detection. Three signal quality assessment (SQA) methods, i.e., Sun's jSQI (2006), a modified jSQI (jSQI2) and Gaussian Template Matching (GTM), were performed and the onset detection results were compared with and without each SQA. For the clean set-p database, the algorithm achieved an accuracy of $99.56 \%$ without SQA and slightly enhanced its accuracy to $99.97 \%, 99.84 \%$ and $99.79 \%$ when using the jSQI, jSQI2 and GTM methods respectively. For the noisy set-p2 database, the algorithm achieved an accuracy of only $76.42 \%$ without SQA but significantly increased to $96.73 \%, 90.60 \%$ and $90.79 \%$ respectively. The jSQI2 and GTM methods exhibited a higher accuracy for assessing the ABP signal quality compared to the jSQI method. In summary, the open-source pulse onset detection algorithm was found to achieve high detection accuracy in a low noise pulsatile database while relative low detection accuracy was observed when using a relatively noisy database. Combining the algorithm with an appropriate SQA procedure significantly improved beat detection accuracy.
\end{abstract}

\section{Introduction}

Pulsatile signals such as arterial blood pressure (ABP) and photoplethysmograph (PPG) contain rich information about the cardiovascular system and can be used to monitor the cardiac activity and verify electrocardiogram (ECG)based alarms for patients in an intensive care unit (ICU)
[1]. Automatic pulse onset detection is a fundamental stage for the beat-level analysis of pulsatile signals. With the onset of each pulse first identified, many other features, as well as the useful clinical parameters, can be calculated and derived, such as slope, pulse peak, pulse amplitude, pulse transit time (PTT), pulse wave velocity (PWV), etc [2]. These features and parameters can be further used for arrhythmia detection, blood pressure and cardiac output estimation, respiration rate estimation and vascular assessment [3].

Accurate and robust detection of pulse onset can be achieved on clean pulsatile signals $[4,5]$. However, in an active clinical environment where noise and artifacts are inevitable, pulse onsets can be easily blurred by noise and motion artifacts due to their intrinsically small amplitude. Thus, accurate and robust pulse onset detection is challenging in a noisy environment.

The open-source algorithm 'wabp.c' proposed by Zong et al. [6] from www.physionet.org has been widely used for clinical applications. This algorithm was subsequently modified by Li and Clifford [7] with a time and amplitude threshold adjustment by changing the slope width of pulse rising edge from 130 to $170 \mathrm{~ms}$ and extending the eyeclosing period after each detection from 250 to $340 \mathrm{~ms}$, to avoid possible double detections in a beat cycle. Since there is no formal validation of this modified algorithm, this study attempts to do so in the context of noisy data.

\section{Methods}

\subsection{Data}

A total of 173 synchronously recorded ECG and ABP pulsatile signals from the PhysioNet/Computing in Cardiology Challenge 2014 were used [8]. These recordings were from two databases (100 from set-p and 73 from setp2). Recordings in set-p had relatively high signal quality (SQ) and thus were used as the clean database, whereas the SQ in recordings from set-p2 was relatively poor and setp2 was therefore used as noisy database. Signals had a duration of 10 minutes and had a varied sample rate between 120 and $1,000 \mathrm{~Hz}$. Manually annotated QRS locations in 
ECG signals were provided as the reference benchmarks for pulse onset detection.

\subsection{Signal quality assessment}

Some ABP pulsatile signals, especially from the set-p2 database are very noisy, resulting in the pulse onsets are almost impossible to be identified by visual inspection. It is therefore reasonable to perform a signal quality assessment (SQA) prior to analysis.

Sun et al. [5] described an open-source method ('jSQI') that tends to capture high energy noise and would invalidate a beat if neighboring beats have low SQ. It is therefore overly sensitive for identifying poor quality beats. Johnson et al. [9] subsequently modified the jSQI code to address these issues (termed jSQI2 here).

In this study, in addition to jSQI and jSQI2, we propose a new method for assessing the ABP signal quality based on Gaussian Template Matching (GTM). For GTM method, we firstly generated four hand-crafted typical pulse templates as shown in Figure 1. Each template, $f(n)$, consisted of three positive Gaussian functions $\left(f_{1}^{*}(n), f_{2}^{*}(n)\right.$ and $\left.f_{3}^{*}(n)\right)$ defined as $[10,11]$ :

$$
\begin{aligned}
& f(n)=\sum_{k=1}^{3} f_{k}^{*}(n) \\
& f_{k}^{*}(n)=H_{k} \times \exp \left(-\frac{2 \times\left(n-C_{k}\right)^{2}}{W_{k}^{2}}\right)
\end{aligned}
$$

where $H_{k}$ denotes the Gaussian peak amplitude, $C_{k}$ denotes the Gaussian peak position, $W_{k}$ denotes the Gaussian half-width and $n$ is the sample index (running from 1 to 1000). The amplitude of template $f(n)$ is normalized to be 1 . The parameter settings for each Gaussian function for each of the four archetypical pulse templates are given in Table 1.

The GTM SQ was then determined by calculating the maximum correlation (MaxCor) between each detected pulse and the four templates. Single beat pulse was regarded having poor SQ if MaxCor meets one of the following two conditions:

Condition 1: MaxCor $<55 \%$

Condition 2: MaxCor $<80 \%$ and (meets A1 or A2)

where A1: pulse amplitude $<20 \mathrm{mmHg}$ and $\mathrm{A} 2$ : maximum of the pulse derivative signal $>10 \mathrm{mmHg}$.

\subsection{Pulse onset detection algorithm}

The open-source pulse onset detection algorithm consisted of three components: a low-pass filter, a windowed and weighted slope sum function, and a decision rule module [6]. First, a second order recursive filter with a $3 \mathrm{~dB}$ cut-off frequency of $16 \mathrm{~Hz}$ was used to suppress high frequency noise [12]. Then a slope sum function (SSF) was calculated by summing the difference signal of the filtered ABP pulse within a fixed time window. This SSF signal was used to enhance the upslope of ABP pulse, as well as
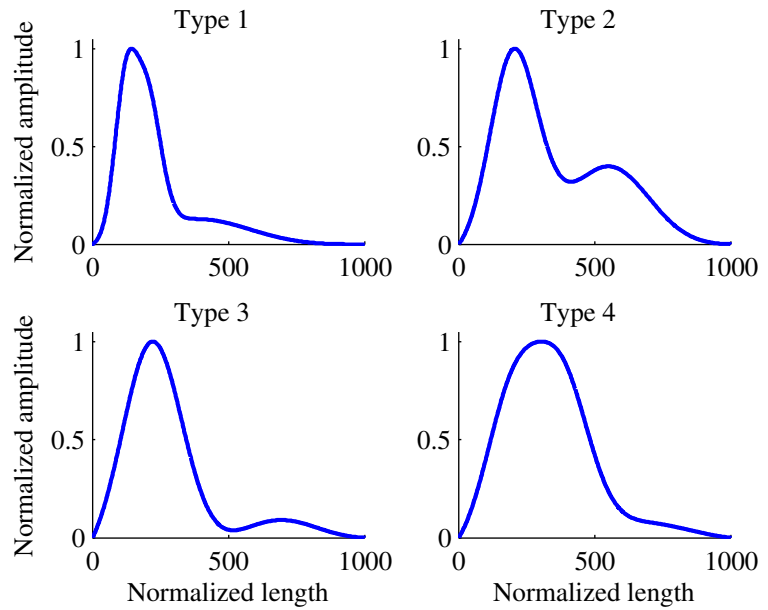

Figure 1. Archetypical ABP pulse templates for the GTM SQ method.

\begin{tabular}{ccccc}
\hline Parameter & \multicolumn{4}{c}{ Pulse template } \\
& Type 1 & Type 2 & Type 3 & Type 4 \\
\hline$H_{1}$ & 0.7 & 0.7 & 0.8 & 0.6 \\
$H_{2}$ & 0.7 & 0.05 & 0.01 & 0.6 \\
$H_{3}$ & 0.15 & 0.3 & 0.1 & 0.1 \\
$C_{1}$ & 120 & 200 & 220 & 200 \\
$C_{2}$ & 200 & 320 & 500 & 380 \\
$C_{3}$ & 380 & 550 & 680 & 650 \\
$W_{1}$ & 80 & 170 & 220 & 200 \\
$W_{2}$ & 100 & 150 & 400 & 200 \\
$W_{3}$ & 400 & 300 & 300 & 400 \\
\hline
\end{tabular}

Table 1. Parameter settings for the Gaussian functions for each type of pulse template.

to suppress the remainder of the ABP waveform. The fixed time window was set as $130 \mathrm{~ms}$ in Zong et al.'s work [6] and was subsequently modified to $170 \mathrm{~ms}$ by Li and Clifford [7]. The onsets of the SSF pulse coincide with the onsets of the ABP pulse and the SSF signal is simple to process. Thus, the pulse onsets were detected from the SSF pulse by a decision rule module. First, an adaptive thresholding method was applied to the SSF signal to detect the appropriate amplitude and then a local search strategy for identifying the likely pulse onsets was employed. Finally, to avoid double detection on the same pulse beat, a 250 $\mathrm{ms}$ eye-closing period was applied, during which the new detected pulse onset was rejected. This eye-closing period was changed to $340 \mathrm{~ms}$ by Li and Clifford [7].

\subsection{Algorithm evaluation}

Let $x_{1}, x_{2}, \cdots, x_{N}$ denote the reference QRS positions. For each position $x_{i}$, the numbers of detected pulse onsets within two time regions: $\left[x_{i}, x_{i}+\delta\right]$ and $\left(x_{i}+\delta, x_{i+1}\right)$ 
were recorded. The detected pulse onsets were expected to appear in the former region. Parameter $\delta$ is a tolerance for determining the true positive $(T P)$, false positive $(F P)$ and false negative $(F N)$ detections and was set as $0.6 \times$ $\left(x_{i+1}-x_{i}\right)$ in this study. The numbers of TP, FP and $F N$ detections were counted and the evaluation metrics of sensitivity $(S e)$, positive predictivity $\left(P_{+}\right)$and accuracy (Acc) were calculated in the standard manner as:

$$
\begin{aligned}
& S e=T P /(T P+F N) \times 100 \% \\
& P_{+}=T P /(T P+F P) \times 100 \% \\
& A c c=T P /(T P+F N+F P) \times 100 \%
\end{aligned}
$$

\section{Results}

As shown in Table 2, for the clean set-p database, the open-source pulse onset detection algorithm without SQA accurately detected $72,132(T P)$ pulse onsets among a total of 72,313 beats, falsely detected $141(F P)$ extra pulse onsets and missed $181(F N)$ actual pulse onsets, which produced an $A c c$ measure of $99.56 \%$. We also observed that SQA procedures filtered the potential incorrect onset detection pulse beats with poor SQ and thus improved the detection accuracy. The numbers of filtered pulse beats were 3,381 for jSQI but only 502 for jSQI2 and 203 for GTM respectively, demonstrating that jSQI is considerably more aggressive in beat removal. For this clean data, the pulse onset detection algorithm with SQA produced slightly higher Acc measures than without SQA, with improvements going from $99.56 \%$ to $99.97 \%, 99.84 \%$ and $99.79 \%$ respectively for the three SQA procedures.

The use of SQA generated more obvious effect on the noisy set-p2 database. Without SQA, the pulse onset detection algorithm truly detected 49,482 (TP) pulse onsets but falsely detected 7,532 (FP) extra pulse onsets and missed 7,740 $(F N)$ actual pulse onsets, which produced a low $A c c$ measure of $76.42 \%$. By contrast, Acc measures were significantly improved to $96.73 \%, 90.60 \%$ and $90.79 \%$ when performing jSQI, jSQI2 and GTM SQA methods respectively. However, the number of filtered pulsatile beats by the SQA procedures was also large. They were as many as 21,303 (accounting for 37\%) removed for jSQI, 14,658 (26\%) removed for jSQI2 and 13,346 (23\%) removed for the GTM method. The highest Acc result reported by the jSQI method is due to the significantly larger number of the removed pulse beats by this method creating a bias towards far easier detections.

Figure 2 shows a SQA and onset detection example for an ABP pulse signal from recording 2850 in set-p2. Three SQA methods, i.e., jSQI, jSQI2 and GTM, were used for assessing the ABP pulse signal quality. The wabp code modified by Li and Clifford [7] was used for detecting the pulse onsets. The upper panel shows the ECG signal (reference QRS positions are marked as red circles) and the lower three panels shows the corresponding SQA and on- set detection results for ABP pulse (detected pulse onsets are marked as pink circles). The shaded areas show the expected area of detected onsets and the solid colours indicate poor SQ (black), a TP detection (green), and a FP and/or a $F N$ detection (red). It is clear that jSQI method tends to report poor SQ beats even though the pulse beat has little noise component. jSQI2 and the new GTM methods give more accurate SQA results than jSQI. In addition, the fourth beat has incorrect $F P$ onset detection and thus it is labelled as red indicator.
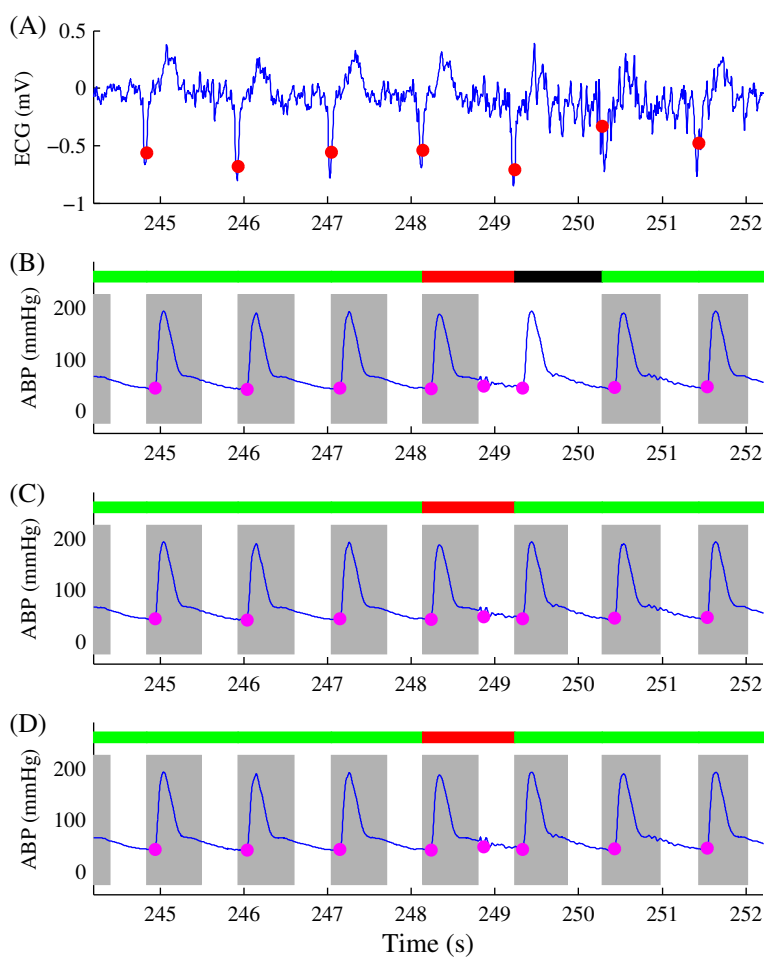

Figure 2. Example of SQA and onset detection results for ABP signal from recording 2850. The upper panel (A) shows the synchronous ECG signal and three lower panels show ABP signal using different SQA methods: (B) jSQI, (C) jSQI2 and (D) GTM. Reference QRS positions are marked as red circles $(\bullet)$ and the detected ABP pulse onsets marked are marked as pink circles $(\bullet)$. The shaded areas indicate the region of detected onsets. Three flag indicators at the top of pulse signal indicate the SQ results for each beat pulse: a poor SQ beat (black), a TP detection (green), and a $F P$ and/or a $F N$ detection (red). Note that jSQI incorrectly labels the beat as noisy.

\section{Discussion and conclusion}

In this study, we tested an open-source pulse onset detection algorithm on both clean and noisy ABP pulsatile signals. The results showed that the tested algorithm could 


\begin{tabular}{lcccccccc}
\hline Variable & \multicolumn{4}{c}{ Clean data (set-p) } & \multicolumn{4}{c}{ Noisy data (set-p2) } \\
& Without SQA & jSQI & jSQI2 & GTM & Without SQA & jSQI & jSQ2 & GTM \\
\hline \# recording & 100 & 100 & 100 & 100 & 73 & 73 & 73 & 73 \\
\# total onsets & 72,313 & 68,932 & 71,811 & 72,110 & 57,222 & 35,919 & 42,546 & 43,876 \\
\# TP onsets & 72,132 & 68,932 & 71,810 & 72,079 & 49,482 & 35,546 & 42,114 & 43,437 \\
\# FN onsets & 181 & 0 & 1 & 31 & 7,740 & 373 & 432 & 439 \\
\# FP onsets & 141 & 23 & 115 & 123 & 7,532 & 828 & 3,925 & 3,970 \\
Se (\%) & 99.75 & 100 & 100 & 99.96 & 86.47 & 98.96 & 98.98 & 99.00 \\
$P+(\%)$ & 99.80 & 99.97 & 99.84 & 99.83 & 86.79 & 97.72 & 91.47 & 91.63 \\
Acc $(\%)$ & 99.56 & 99.97 & 99.84 & 99.79 & 76.42 & 96.73 & 90.62 & 90.79 \\
\hline
\end{tabular}

Table 2. Results of the tested open-source pulse onset detection algorithm for both clean and noisy databases.

achieve high detection accuracy on the clean data with Acc measure of $99.56 \%$. However, the Acc measure dropped to $76.42 \%$ when evaluated on the noisy data, indicating the strong noise response on the tested algorithm.

We compared the accuracy of the tested algorithm between with and without SQA applied. By using SQA to remove noisy beats, $A c c$ measures were slightly enhanced for the clean data, and greatly enhanced for noisy data. Thus, implementing a SQA procedure is necessary for the pulse onset detection algorithm, especially when processing low quality signals. We also compared the performances of three different SQA procedures and the results showed that the open-source jSQI method tends to identify many high SQ pulse beats as poor SQ beats. jSQI2 and a novel GTM methods were shown to be more accurate for assessing the ABP signal quality than jSQI.

The novelty of the GTM method described here for assessing the ABP pulse signal quality, like the other two methods, does not require a pre-learning process to obtain the pulse templates and can perform the analysis from the first beat. The GTM method has potential utility for use in the SQA of pulsatile signals in real-time environments. Moreover, the creation of a bespoke template on a per-patient basis, during a rapid learning period, and then later adapting over time, provides the potential for a more accurate system, tuned to a variety of waveforms, which do not exhibit natural upper and lower bounds (such as the photoplethysmogram).

\section{Acknowledgments}

Chengyu Liu would like to thank the International Postdoctoral Exchange Programme of the National Postdoctoral Management Committee of China and Emory University for generous funding.

\section{References}

[1] Aboukhalil A, Nielsen L, Saeed M, Mark RG, Clifford GD. Reducing false alarm rates for critical arrhythmias using the arterial blood pressure waveform. Journal of Biomedical Informatics 2008;41(0):442-451.
[2] Xu P, Bergsneidera M, Hu X. Pulse onset detection using neighbor pulse-based signal enhancement. Medical Engineering Physics 2009;31(0):337-345.

[3] Allen J. Photoplethysmography and its application in clinical physiological measurement. Physiological Measurement 2007;28(0):R1-39.

[4] Kazanavicius E, Gircys R, Vrubliauskas A, Lugin S. Mathematical methods for determining the foot point of the arterial pulse wave and evaluation of proposed methods. Information technology and control 2005;34(0):29-36.

[5] Sun JX, Reisner AT, Mark RG. A signal abnormality index for arterial blood pressure waveforms. In Computing in Cardiology, volume 33. 2006; 13-16.

[6] Zong W, Heldt T, Moody GB, Mark RG. An open-source algorithm to detect onset of arterial blood pressure pulses. In Computing in Cardiology, volume 30. 2003; 259-262.

[7] Li Q, Clifford GD. Dynamic time warping and machine learning for signal quality assessment of pulsatile signals. Physiological Measurement 2012;33(0):1491-1501.

[8] The physionet/computing in cardiology challenge 2014. URL www. physionet.org/challenge/2014/.

[9] Johnson AE, Behar J, Andreotti F, Clifford GD, Oster J. Multimodal heart beat detection using signal quality indices. Physiological Measurement 2015;36(0):1665-1677.

[10] Liu CY, Zheng D, Murray A, Liu CC. Modelling carotid and radial artery pulse pressure waveforms by curve fitting with gaussian functions. Biomedical Signal Processing and Control 2013;8(0):449-454.

[11] Liu CY, Zheng D, Zhao LN, Liu CC. Gaussian fitting for carotid and radial artery pressure waveforms: comparison between normal subjects and heart failure patients. Bio medical Materials and Engineering 2014;24(0):271-277.

[12] Lynn PA. Online digital filters for biological signals: some fast designs for a small computer. Medical Biological Engineering Computing 1977;15(0):534-540.

Address for correspondence:

Chengyu Liu

Department of Biomedical Informatics

Emory University

Atlanta, USA

E-mail: chengyu.liu@emory.edu 